\title{
Gear diagnostics in a planetary gearbox: a study using internal and external vibration signals
}

\author{
W Smith, L Deshpande, R Randall and Huaizhong Li \\ Paper presented at CM 2013 \& MFPT 2013, The Tenth International Conference on Condition Monitoring and Machinery Failure Prevention \\ Technologies, Kraków, Poland, June 2013
}

This paper investigates gear diagnostics using laboratory measurements on a specially-designed planetary gearbox test-rig. Planetary gearboxes present a unique challenge to the condition monitoring community. They are complex units, comprising many rotating components, and any vibration signal arising from a faulty gear or bearing, as measured on the outer casing, is likely to contain a substantial amount of extraneous information.

While a number of signal processing techniques have been developed in attempts to overcome these difficulties, in this paper we investigate the potential of using an internallymounted accelerometer to give improved diagnostic performance in the case of seeded planet gear faults. The vibration is also measured on the outside of the gearbox casing and the diagnostic potential of the two measurements is compared. It is found that in most cases the internal measurements actually give inferior diagnostic performance, although some problems with the internal signals preclude a complete comparison.

Along with the measurement points, two diagnostic procedures are compared, both based on the kurtosis of the residual signal obtained after removing regular gear meshing components. The first is based on the well-known FM4 parameter, which uses time synchronous averaging to establish the regular meshing pattern. The second focuses on kurtosis enhancement using autoregressive linear prediction filtering followed by spectral kurtosis. The latter method was found to greatly increase the detection capability, with very high kurtosis values obtained in most fault cases.

\section{Introduction}

Due to their compact nature and ability to handle large torque loads, planetary gearboxes are a common feature in a number of machines, in particular helicopter and wind turbine transmissions. From a condition monitoring viewpoint, however, they pose a unique challenge. They are complex units, with many rotating parts, and there is restricted access to the various internal components.

The application of vibration-based diagnostic techniques to such gearboxes is complicated by the fact that, for many common faults, the transmission path between the fault signal source and any fixed measurement point - the outer casing, for example - is

Wade Smith, Lav Deshpande, Robert Randall and Huaizhong Li are with the School of Mechanical and Manufacturing Engineering, University of New South Wales, Sydney, NSW, 2052, Australia. Tel: +61 29385 4121; Fax: +61 29663 1222; Email: wade.smith@unsw.edu.au time-varying. Moreover, the required transmission path is often not very direct. These complications mean that the fault signal is subject both to modulation and to considerable contamination by noise, as measured at some fixed external point.

In attempts to overcome these difficulties, a number of signal processing techniques have been developed - generally comprising various forms of windowing, such as that proposed by McFadden ${ }^{[1]}$, or signal weighting, as suggested by Forrester ${ }^{[2]}$ - which allow the application of conventional diagnostic techniques, such as time synchronous averaging (TSA).

Recently, de Smidt ${ }^{[3]}$ proposed and applied a novel approach for planetary gearbox diagnostics, which involved the analysis of not only the externally measured vibration, but also the vibration as measured on the planet carrier, internal to the gearbox. The latter case required the permanent mounting of an accelerometer on the planet carrier and a slip ring to transmit the signal from the rotating transducer. The study found that the internal measurements gave comparable, and in some cases superior, diagnostic capability in the case of seeded planet gear faults. While the idea of an accelerometer mounted on the planet carrier may seem unsuitable in practical applications, de Smidt argued that, although it may not be so feasible now, that would be likely to change in the future, in particular with improvements in wireless technologies ${ }^{[3]}$.

The authors recently applied this internal vibration approach to planetary gearbox bearing diagnostics ${ }^{[4]}$. It was found that the internal vibration measurements gave improved diagnostic performance in the presence of localised inner race faults seeded on the planet gear bearings, while in the case of outer race faults the internal and external measurements provided similar diagnostic outcomes.

The focus of the present paper is on seeded planet gear faults, the objective being to extend de Smidt's work ${ }^{[3]}$ by looking at a broader range of fault types and conditions. While de Smidt investigated mostly planet gear spalls in mesh with the ring gear, the present study investigates a half-tooth fault, a tooth root crack and a tooth spall, the last two of which are studied in both possible meshing configurations (planet-ring and planetsun). The inclusion of the first two fault types is an important extension because they are stiffness- rather than geometric-based, and so will generally give a different vibration signature. The use of faulty planet-sun (rather than only planet-ring) mesh pairs is also an important extension, because it is generally thought that planet-sun mesh faults are more difficult to detect than those of the planet-ring - at least using a traditional fixed external accelerometer - yet are equally important from a condition monitoring point of view. 
The studied measurements were obtained from a speciallydesigned gearbox test-rig at the University of New South Wales (UNSW). In each gear fault scenario, the vibration signals from both internal and external measurement points are compared and the signal processing steps required to achieve a satisfactory diagnosis are discussed. Indeed, a secondary contribution of the paper is a comparison in performance of the traditional gear diagnostic approach based on time synchronous averaging, with an alternative approach based on autoregressive linear prediction filtering and spectral kurtosis.

The main objective of the study is to gain a further insight into the possible limitations of external vibration-based gear diagnostics in planetary gearboxes and to investigate what might be gained by using internal vibration measurements. The paper makes a number of observations and recommendations in that regard.

\section{Experimental set-up}

\subsection{Gear arrangement}

Figure 1 shows the basic layout of a planetary gearbox, for which there are many possible gearing arrangements. In the gearbox used in this study, the ring gear was fixed, the planet carrier, connected to three equi-spaced planet gears, provided the input, and the sun gear provided the output, with all gears of the conventional spur type.

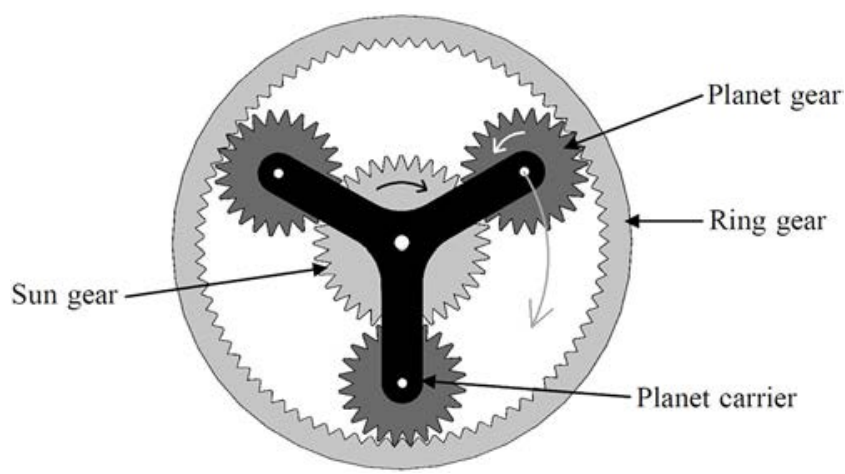

Figure 1. Basic layout of planetary gearbox ${ }^{[5]}$

The gearbox was installed on the UNSW gear-rig, which also included an initial parallel gear reduction stage. The arrangement of both gear stages is shown in Figure 2, where it can be seen that the numbers of teeth on the ring gear, sun gear and planet gear, respectively, were 80,40 and 20 . This arrangement - far from best-practice 'hunting tooth' design - was necessary due to space limitations, commercial gear availability and the need to maintain close to a 1:1 overall ratio on the rig. The chosen arrangement provides a 1:3 increase through the planetary stage and an overall ratio of $15: 16$.

\subsection{Test-rig}

In addition to the gearbox, the UNSW gear-rig consists of an AC induction motor with variable speed controller, a recirculating hydraulic system that provides a resistance torque and two large flywheels to reduce speed fluctuations, all connected through shafts with a number of flexible couplings to mitigate shaft misalignment.

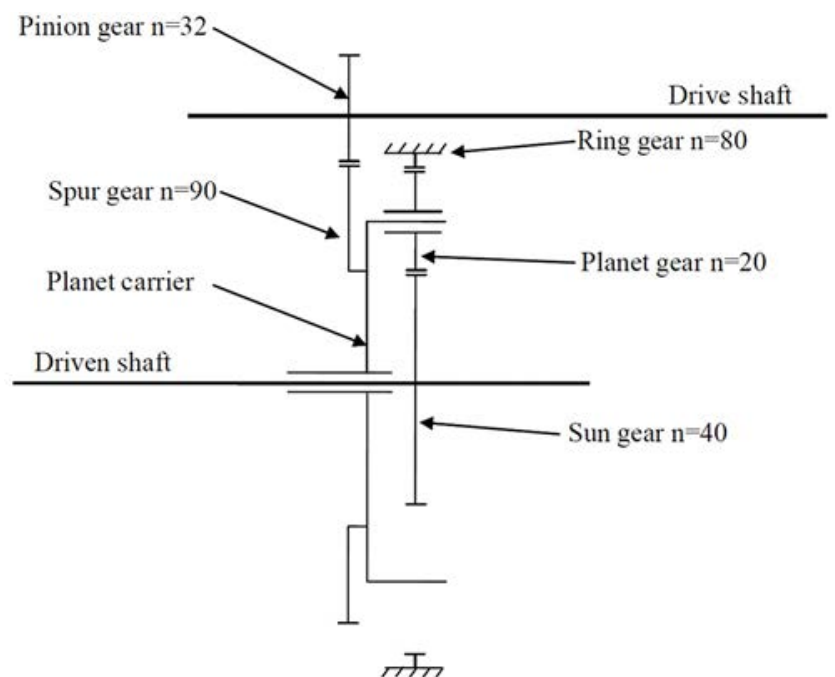

Figure 2. Gear details for the UNSW planetary gearbox testrig $^{[6]}$

\subsection{Seeded gear faults}

To simulate various types of gear damage, three types of seeded planet gear faults were investigated in this study. The first was simply a half-tooth fault, achieved by removing material of one tooth on one planet gear to reduce it to half its original width. The second was a tooth root crack, achieved by machining a $45^{\circ}$ angle slot of approximately $0.5 \mathrm{~mm}$ width across the whole tooth at the tooth root. The slot was extended such that it met the radius drawn to the tooth centre. The third investigated fault was a tooth spall, seeded by machining a channel of $1 \mathrm{~mm}$ width and $0.1 \mathrm{~mm}$ depth across the tooth at the pitch line. All of the studied gear faults can be seen in Figure 3.

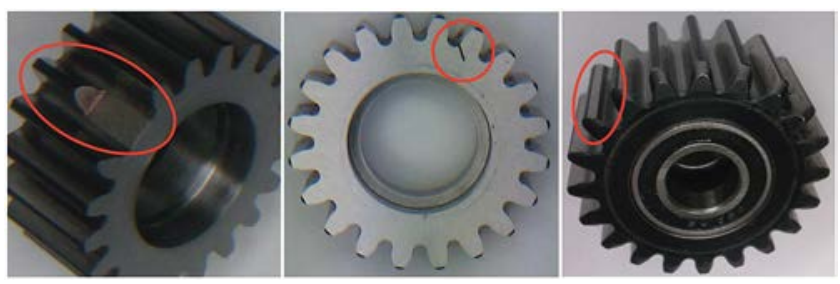

Figure 3. Seeded planet gear faults. Left: half tooth; middle: tooth root crack; right: tooth spall

\subsection{Instrumentation and data acquisition}

The internal and external vibration was measured on the gearbox using Brüel \& Kjær 4394 IEPE-type and 4393 charge-type accelerometers, respectively. The internal accelerometer was stud-mounted in the axial direction on the planet carrier and the signal was transmitted via a Michigan Scientific B6-2 slip ring. The external accelerometer was mounted radially on the top of the outside of the ring gear, the idea being to replicate the closest measurement point that might be feasible in a more conventional diagnostic environment.

The two accelerometers, along with a once-per-revolution tachometer mounted on the output shaft, were connected to a Brüel \& Kjær PULSE analyser. For each test, a record length of $20 \mathrm{~s}$ and a sample rate of $65,536 \mathrm{~Hz}$ were used.

Figure 4 shows the placement of the transducers from a top view of the gearbox (internal accelerometer not shown). 


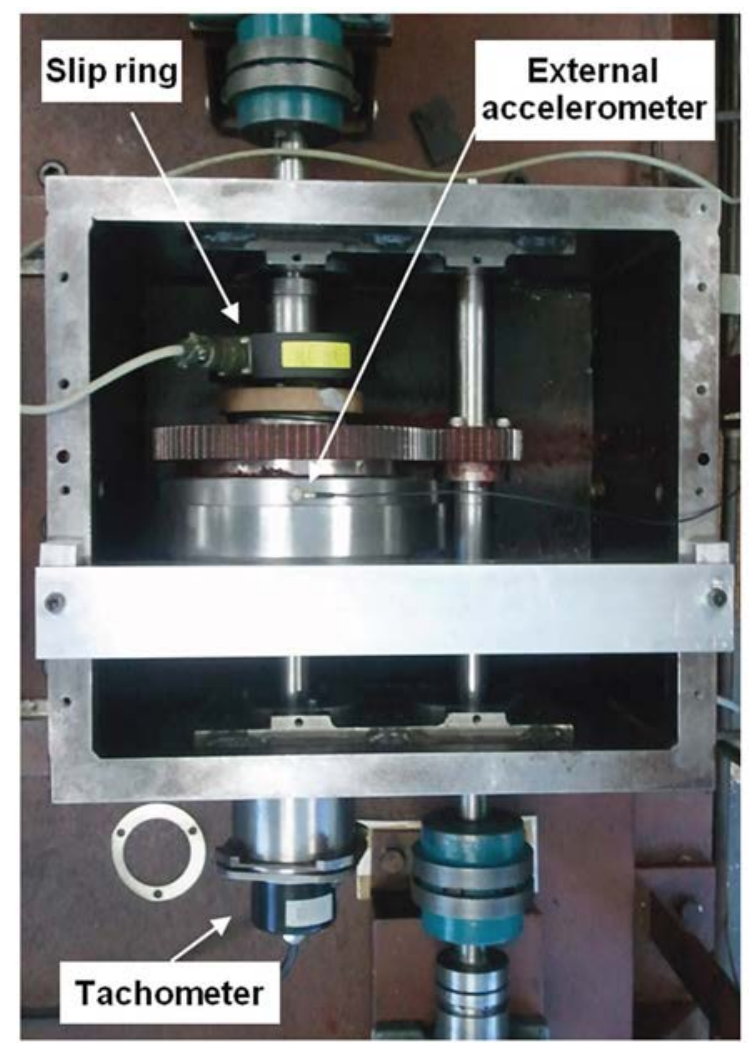

Figure 4. Top view of UNSW planetary gearbox showing transducer placement

\subsection{Test programme and conditions}

Each test was conducted with one of the faulty planet gears installed in the gearbox, and one fault-free test was conducted as a baseline case. For the half-tooth case, because it gives a theoretically identical stiffness reduction (of 50\%) for both planet-ring and planet-sun meshes, only one test was carried out. For the root crack and tooth spall, however, two tests were carried out, since the effect of these faults - stiffness-based for the former and geometric for the latter - depends on which side of the planet gear teeth mesh with the ring or the sun. Thus, tests were carried out with the faulty planet gear flipped to reverse the planet-ring/ planet-sun meshing relationship. All the tests are summarised in Table 1, which also includes the approximate tooth mesh stiffness reduction for each fault. For the root cracks, the stiffness reduction was estimated using a simple two-dimensional finite element gear model and applying a static force at the pitch point, at an assumed pressure angle of $20^{\circ}$. It should be noted that the seeded root crack was of sufficient width $(0.5 \mathrm{~mm})$ to allow the tooth to deflect freely in both directions without the crack completely closing - perhaps different to most naturally occurring tooth cracks.

The tests were conducted at a nominal input shaft speed of $6 \mathrm{~Hz}$ (planet carrier speed of $f_{c}=2.13 \mathrm{~Hz}$ ) and a constant torque load of $50 \mathrm{Nm}$. This meant the planet gears were driven at only about $20 \%$ of their rated torque load (in bending). It was not possible to test at a higher load due to the allowable torque of the drive pinion in the parallel gear stage. The ring gear was located through a bracket mounted via bearings on the output shaft, thus ensuring the sun, planet carrier and ring were all concentric. The
Table 1. Gear fault descriptions

\begin{tabular}{|l|l|}
\hline Test name & Gear fault description \\
\hline No fault & Baseline case with no gear fault \\
\hline Half tooth & $\begin{array}{l}\text { Half-tooth fault - 50\% reduction in planet-ring and } \\
\text { planet-sun mesh stiffness }\end{array}$ \\
\hline Tooth crack A & $\begin{array}{l}\text { Tooth root crack opening when meshing with the } \\
\text { sun - reduction in mesh stiffness of 66\% for planet- } \\
\text { ring and 57\% for planet-sun }\end{array}$ \\
\hline Tooth crack B & $\begin{array}{l}\text { Tooth root crack opening when meshing with the } \\
\text { ring - reduction in mesh stiffness of 57\% for planet- } \\
\text { ring and 66\% for planet-sun }\end{array}$ \\
\hline Tooth spall A & $\begin{array}{l}\text { Tooth spall meshing with the sun - geometric fault } \\
\text { with negligible change in mesh stiffness }\end{array}$ \\
\hline Tooth spall B & $\begin{array}{l}\text { Tooth spall meshing with the ring - geometric fault } \\
\text { with negligible change in mesh stiffness }\end{array}$ \\
\hline
\end{tabular}

ring gear was constrained to rotate by a holding bracket attached to the gearbox casing, as can be seen in Figure 4. Although a tachometer was used on the output shaft, no tooth numbering or angular referencing was used to relate the gear faults to the tachometer pulses. This situation reflects more closely what would be experienced in practice.

\section{Signal processing methodology}

Two basic approaches were used to process the measured signals, both commencing with order tracking to remove the effect of speed fluctuations. The regular gear meshing components were then removed from the signal with two different approaches and the kurtosis of the residual signal was calculated, this being a measure of signal impulsivity and hence an indicator of possible damage. Finally, envelope analysis of the residual signal was conducted to determine the repetition frequencies of any impulsive events.

\subsection{Procedure 1: Time synchronous averaging}

The complete process for the first analysis procedure is shown in Figure 5. It is based on Stewart's FM4 parameter, which is defined

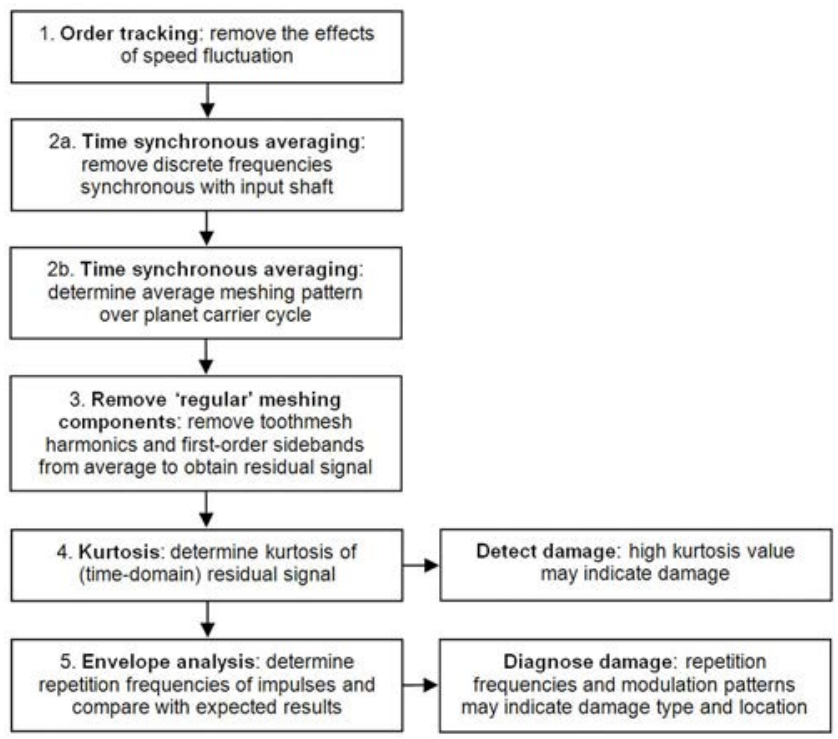

Figure 5. Procedure 1: Time synchronous averaging 
as the kurtosis of the time synchronous average (TSA) signal after removal of the regular gear meshing components ${ }^{[7]}$, which in this case is achieved by removing the tooth mesh harmonics and first-order sidebands. The components synchronous with the input shaft (ie the parallel gear stage) are removed with an initial stage of averaging.

In the planetary stage of TSA, the signal is averaged over one planet carrier cycle and thus represents a composite of the vibration from all planet gears. The non-hunting tooth design of this gearbox means that all gear pair meshes occur at the same spatial point in each planet carrier cycle, which involves exactly one planet-ring meshing cycle and two planet-sun meshing cycles. This makes techniques focused on the averaging of single meshing pairs - such as McFadden's windowing method ${ }^{[1]}$ and Forrester's signal weighting approach ${ }^{[2]}$ - difficult to apply. These difficulties prompted the use of a second signal processing approach, as outlined in the next section.

\subsection{Procedure 2: Autoregressive linear prediction filtering and spectral kurtosis}

The second procedure used to remove regular gear meshing components was autoregressive (AR) linear prediction filtering, similar to that proposed by Wang and Wong ${ }^{[8]}$. This approach predicts the value of the signal at a given time by using a linear combination of prior values. Wang and Wong applied the technique to gear diagnostics and determined the filter coefficients based on baseline (undamaged) data, but in this study we apply the filter directly to each signal. This approach was applied to bearing diagnostics by Sawalhi et al ${ }^{[9]}$ and is used here with the idea that, assuming the right filter length, the model will be able to predict regular meshing patterns but will be unable to predict the impulses arising from gear faults. Thus, the impulses are left in the error, or residual, signal, which is then studied further. The only parameter the user need specify with this approach is the filter length. Here, the same criterion as that proposed by Sawalhi et al is used: the best filter length is assumed to be that which maximises the kurtosis of the residual signal. The only other stipulation on filter length was that it did not exceed the period between expected fault impulses, so, to satisfy that requirement and to reduce computation time, a

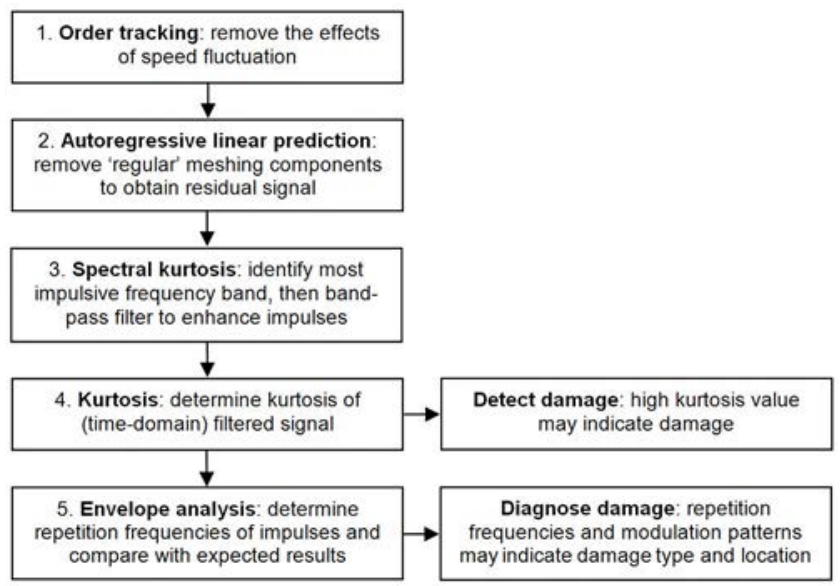

Figure 6. Procedure 2: AR linear prediction filtering and spectral kurtosis maximum length of one quarter of a fault separation period was imposed.

Spectral kurtosis (SK) was then applied to the residual signal to identify the band of maximum impulsivity ${ }^{[10]}$. The SK was determined using the computationally-efficient 'fast kurtogram', as developed by Antoni ${ }^{[11]}$.

\subsection{Other signal processing approaches}

Overall, the second signal processing approach (Section 3.2) seems better suited than the first (Section 3.1) to this application. Given the difficulty in forming a TSA signal for the individual planet gears (rather than a composite average, as used here), techniques based directly on the TSA signal are less likely to prove successful. For example, amplitude and phase demodulation of the tooth mesh harmonics ${ }^{[12]}$ were tried but did not yield any useful results. This is not surprising given the large number of meshes (six) occurring in the planetary stage, and is probably further compounded by the gearbox having 'sequential mesh phasing' ${ }^{[13]}$, meaning that each planet gear has a mesh cycle that is $120^{\circ}$ out of phase with those of the other two.

It makes sense, then, to use a flexible approach to remove the regular gear meshing components and to place a greater emphasis on kurtosis enhancement. Indeed, the recent application of spectral kurtosis to one planetary gearbox failure was found to provide a much more advanced warning than established techniques $^{[14]}$.

\subsection{Expected gear fault signals}

For each planet carrier cycle, the seeded gear faults are expected to produce the following impulses:

- Half-tooth fault: eight impulses, with two groups of four having similar characteristics, each group corresponding to the planet-ring or planet-sun meshes. Although the mesh pair stiffness reduction is identical for both groups, the smaller contact ratio for the planet-sun means that a given stiffness reduction has a larger overall effect than for the planet-ring.

- Tooth root crack: eight impulses, with two groups of four having similar characteristics, owing both to the difference in planet-ring and planet-sun mesh stiffnesses and also to the difference in contact ratio between the two groups.

- Tooth spall: four similar-sized impulses, since the fault only meshes with the ring or the sun.

In each case, the impulses occurring at either the planet-ring or planet-sun meshes are expected to be equi-spaced in the angledomain record. However, for the half-tooth and root crack cases - faults that interact with both ring and sun - a difference in mesh phasing between each planet's ring and sun meshes means that the two series of impulses (from interaction with the ring and the sun) are slightly offset from one another and, thus, are not exactly equi-spaced in the angle domain.

Note that the signal measured at the external accelerometer would also be subject to modulation by the planet carrier frequency, $f_{\mathcal{c}}$, due to the varying transmission path between the fault and the transducer, which would not be evident in the case of the internal accelerometer. 


\section{Results and discussion}

\subsection{Damage detection using kurtosis}

\subsubsection{Raw signal results}

Figure 7 shows the kurtosis values of the raw signals, calculated prior to any processing. Note that all of the kurtosis figures in this paper are given as the normalised fourth order moment without subtraction of 3 - the kurtosis of an ideal Gaussian distribution. Thus, the 'no fault' case here would be expected to have a kurtosis of around 3, and the trigger setting indicating damage might be set to around 4.5 or 5 . Figure 7, then, shows that without signal processing most of the studied faults would go undetected, the main exception being the external measurement of 'tooth spall $\mathrm{A}$ ' (tooth spall in mesh with the sun). Also of note is that in the cases where the fault would be detected - 'tooth spall A' and possibly 'tooth crack B' - the external measurement gives a higher kurtosis than the internal one.

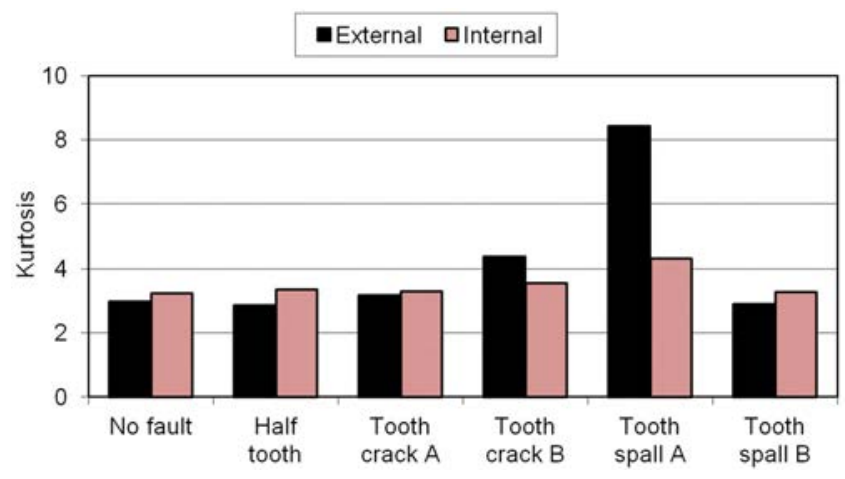

Figure 7. Kurtosis of raw signals

\subsubsection{Procedure 1 (TSA) results}

Figure 8 shows the kurtosis values of the signals processed with Procedure 1 (Section 3.1). Overall, it can be seen that little has changed from the raw signal results and most faults would still go undetected. It is perhaps surprising that, based on these results, the internal measurements would fail to diagnose any of the faults.

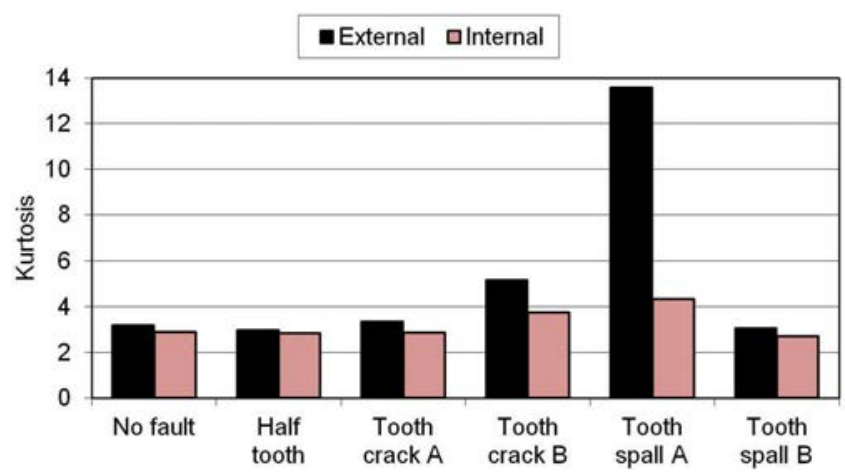

Figure 8. Kurtosis of signals processed using Procedure 1 (TSA)

\subsubsection{Procedure 2 (AR and SK) results}

Figure 9 shows the kurtosis values of the signals processed with Procedure 2 (Section 3.2) and it can be seen that a dramatic increase has occurred in the kurtosis of most of the signals. The results suggest that the external measurements would easily detect all of the fault cases except for the half tooth. The results for the internal measurements, however, are mixed and require some explanation.

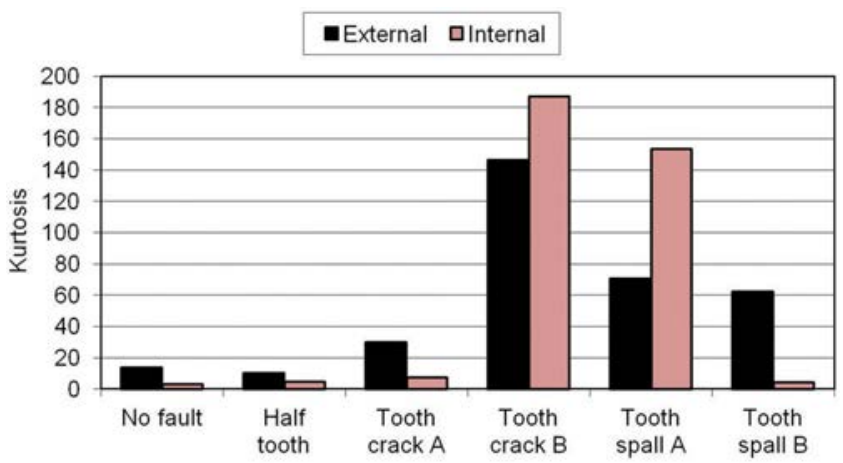

Figure 9. Kurtosis of signals processed using Procedure 2 (AR and SK)

After testing it was noticed that the acceleration signals from the internal accelerometer were contaminated by electrical interference and dropouts. The interference was identified as coming from the variable speed motor controller and also from a ground loop effect, while the dropouts served to give an artificially high kurtosis. In another study, on bearing diagnostics, the authors were able to overcome this problem by strategically selecting a 'relatively uncontaminated' demodulation band for envelope analysis ${ }^{[4]}$. This was again attempted here, although it appears the results are mixed. In the cases of 'tooth crack B' and 'tooth spall A' the internal measurements give very high kurtosis values, but with the other faults the kurtosis is substantially lower than that found with the external measurements. This is likely to be due to the fact that the most impulsive frequency bands were contaminated by electrical noise and so a suboptimal band was selected for investigation. It should be noted, however, that this contamination does not affect the results in Section 4.1.2, which are based on the TSA signal comprising only discrete frequency components at harmonics of the planet carrier.

\subsection{Damage diagnosis using envelope analysis}

Space limitations preclude the inclusion of the envelope spectra here, but in general they were found to not give a reliable diagnosis of damage. That is because most envelope spectra were found to be dominated by planet carrier harmonics, yet, due to the non-hunting tooth design of this gearbox, all expected fault frequencies and modulation sidebands are integer multiples of the planet carrier frequency. Nevertheless, the envelope spectra for 'tooth spall A' gave good results with both processing approaches and both measurement points, so perhaps envelope analysis would have been more successful had the other faults been more severe.

\subsection{Further discussion}

Due to the abovementioned problems with the internal acceleration signals, it is difficult to give a conclusive assessment of the relative performance of the two measurement points, although it is interesting to note that in the TSA-based approach, which negated the internal signal problems, the external accelerometer 
gave superior detection performance, easily detecting 'tooth spall A' and giving a borderline detection of 'tooth crack B'.

Also of note is that tooth spalls were consistently easier to detect when meshing with the sun rather than the ring. This seems counterintuitive, perhaps indicating a dominant transmission path through the sun gear in this gearbox. Indeed, Lewicki et al found planet tooth spalls very easy to detect in both mesh configurations, and they reported that faulty planet-sun meshes resulted in "leakage of the defect signal to the neighbouring planets" ${ }^{[15]}$. Further studies and numerical simulations aim to confirm these findings.

\section{Conclusions}

This paper investigated gear diagnostics in a planetary gearbox using external and internal vibration measurements from a laboratory test-rig. It was found that in most cases the external acceleration measurements provided superior damage detection capability, although due to problems with the internal acceleration signals a conclusive assessment of the relative performance of the two measurement points is difficult to draw.

The gearbox under investigation comprised an extreme non-hunting tooth design, which limited the effective use of conventional time synchronous averaging. Thus, an alternative signal processing approach was employed, based on kurtosis enhancement using autoregressive linear prediction filtering followed by spectral kurtosis. This method was found to greatly increase the detection capability, with all but one fault (half tooth) easily detectable using the external acceleration signal.

One interesting finding of the paper is that planet tooth spalls were much easier to detect when meshing with the sun gear rather than the ring. Further studies and numerical simulations aim to confirm this finding.

\section{Acknowledgements}

The authors wish to thank Andrew Bligh for the initial design of the planetary gearbox. This work was supported in part by the Australian Research Council, through the Linkage Project (LP110200738) and also by Australia's Defence Science and Technology Organisation (DSTO).

\section{References}

1. P D McFadden, 'A technique for calculating the time domain averages of the vibration of the individual planet gears and the sun gear in an epicyclic gearbox', Journal of Sound and Vibration, Vol 144, No 1, pp 163-172, 1991.

2. D B Forrester, 'A method for the separation of epicyclic planet gear vibration signatures', presented at the Third International Conference on Acoustical and Vibratory Surveillance Methods and Diagnostic Techniques, Senlis,
France, 12-15 October 1998.

3. M R de Smidt, 'Internal vibration monitoring of a planetary gearbox', ME Thesis, University of Pretoria, 2009.

4. W Smith, L Deshpande, R Randall and $\mathrm{H} \mathrm{Li}$, 'Bearing diagnostics in a planetary gearbox: a study using internal and external vibration signals', presented at the 26th International Congress of Condition Monitoring and Diagnostic Engineering Management, Helsinki, Finland, 11-13 June 2013.

5. S Vorkoetter, 'Demystifying gearing and gearboxes', www. stefanv.com/rcstuff/qf200003.html, 2000.

6. A Bligh, 'Design, manufacture and testing of a planetary gearbox for the purpose of internal vibration-based condition monitoring', BE Thesis, UNSW, 2012.

7. R M Stewart, 'Some useful data analysis techniques for gearbox diagnostics' (Paper \#18), Proceedings of the Meeting on the Applications of Time Series Analysis, ISVR, University of Southampton, Southampton, UK, 19-22 September 1977.

8. W Wang and A K Wong, 'Autoregressive model-based gear fault diagnosis', Journal of Vibration and Acoustics, Vol 124, No 2, pp 172-179, 2002.

9. N Sawalhi, R B Randall and H Endo, 'The enhancement of fault detection and diagnosis in rolling element bearings using minimum entropy deconvolution combined with spectral kurtosis', Mechanical Systems and Signal Processing, Vol 21, No 6, pp 2616-2633, 2007.

10. J Antoni and R B Randall, 'The spectral kurtosis: application to the vibratory surveillance and diagnostics of rotating machines', Mechanical Systems and Signal Processing, Vol 20, No 2, pp 308-331, 2006.

11. J Antoni, 'Fast computation of the kurtogram for the detection of transient faults', Mechanical Systems and Signal Processing, Vol 21, No 1, pp 108-124, 2007.

12. P D McFadden, 'Detecting fatigue cracks in gears by amplitude and phase demodulation of the meshing vibration', Journal of Vibration, Acoustics, Stress and Reliability in Design, Vol 108, No 2, pp 165-170, 1986.

13. R G Parker and J Lin, 'Mesh phasing relationships in planetary and epicyclic gears', Journal of Mechanical Design, Vol 126, No 2, pp 365-370, 2004.

14. T Barszcz and R B Randall, 'Application of spectral kurtosis for detection of a tooth crack in the planetary gear of a wind turbine', Mechanical Systems and Signal Processing, Vol 23, No 4, pp 1352-1365, 2009.

15. D G Lewicki, K E LaBerge, R H Ehinger and J Fetty, 'Planetary gearbox fault detection using vibration separation techniques', NASA Report NASA/TM-2011-217127, prepared for the American Helicopter Society 67th Annual Forum and Technology Display, Virginia Beach, Virginia, USA, 3-5 May 2011. 